\title{
GaN Thin Films Deposited on n-Si (111) Substrate with a Metal Organic Chemical Vapor Deposition System for Sensing Ultraviolet Light
}

\author{
Tse-Heng Chou \\ Department of Fire Science, WuFeng University, Minhsiung, Chiayi 62153, Taiwan
}

(Received March 13, 2015; accepted March 18, 2016)

Keywords: cubic $\beta$-SiC, porous $\beta$-SiC, rapid thermal chemical vapor deposition, ultraviolet, photo and dark current ratios

Metal organic chemical vapor deposited $\mathrm{GaN}$ thin films on n-Si (111) substrates with cubic $\beta-\mathrm{SiC}$ (c-SiC) and porous $\beta$-SiC (PSC) buffer layers were characterized and compared with each other. We used rapid thermal chemical vapor deposition (RTCVD) to grow the c-SiC layers, and some of them were electrochemically anodized to obtain the PSC layers. Then the deposited GaN films were characterized with X-ray diffraction (XRD), Fourier transform infrared spectroscopy (FTIR), atomic force microscopy (AFM), scanning electron microscopy (SEM), and Raman spectroscopy. To study the GaN thin films for ultraviolet-detecting applications, we measured the photo and dark currents of a metal-semiconductor-metal (MSM) photodiode with and without the irradiance of an ultraviolet (UV) light source $\left(366 \mathrm{~nm}, 6 \mathrm{~mW} / \mathrm{cm}^{2}\right)$. The photo and dark current ratios (PDCRs) of both structures are high at room temperature, especially for the devices on the PSC buffer layer; a value of as high as $6.75 \times 10^{5}$ under $-1 \mathrm{~V}$ bias has been achieved. We attribute the high PDCR to the suppression of the induced stress in the GaN film by the PSC buffer layer.

\section{Introduction}

Commonly, gallium nitride $(\mathrm{GaN})$ thin films are grown heteroepitaxially on either sapphire $\left(\alpha-\mathrm{Al}_{2} \mathrm{O}_{3}\right)$ or single crystal $(\alpha-6 \mathrm{H})$ silicon carbide ( $\left.\mathrm{SiC}\right)$ substrates. ${ }^{(1)}$ However, these substrates have poor thermal conductivity, high cost, and limited size. In addition, $\mathrm{GaN}$ on sapphire has a large $(>12 \%)$ lattice mismatch and deviation of thermal expansion coefficients, ${ }^{(2)}$ which result in a large threading dislocation (TD) density of $\sim 10^{10} \mathrm{~cm}^{-2}$ in the grown GaN thin film. ${ }^{(3,4)}$ The TDs in GaN are electrically active and degrade the transport, emission, and detection properties. ${ }^{(3)}$ Epitaxial $\mathrm{GaN}$ on Si offers an advantage of lower cost than that grown on sapphire or $(\alpha-6 \mathrm{H}) \mathrm{SiC}$. Moreover, the $\mathrm{GaN}$ on Si has a high thermal conductivity and the potential for monolithic integration of GaNbased devices with conventional microelectronics. ${ }^{(1)}$ However, direct growth of a GaN layer on Si substrates also leads to poor morphology owing to a large lattice mismatch and a difference in the thermal expansion coefficient between $\mathrm{GaN}$ and $\mathrm{Si}^{(5)}$ Therefore, an appropriate buffer layer is required for the successful heteroepitaxial growth of $\mathrm{GaN}$ on $\mathrm{Si}$ (111). In the past, different buffer layers such as $3 \mathrm{C}-\mathrm{SiC}(\beta-\mathrm{SiC})$, AlN, AlAS, GaAs, and AlGaN have been investigated. ${ }^{(1,5)}$ The most

${ }^{*}$ Corresponding author: e-mail: thchou@wfu.edu.tw 
promising one among these buffers is $\beta$-SiC owing to its relatively low lattice mismatch $(\sim 3 \%)$ with GaN.(3)

In this study, crystalline GaN films were deposited on n-Si (111) substrates using a metal organic chemical vapor deposition (MOCVD) system with both cubic $\beta$-SiC (c-SiC) and porous $\beta$-SiC (PSC) as buffer layers. Then, we studied the optoelectronic properties of the grown GaN thin films for ultraviolet (UV)-light-detecting applications by measurement of the photo and dark current ratio (PDCR) on a metal-semiconductor-metal (MSM) photodiode (see Fig. 1) with and without the irradiation by a UV light source $\left(366 \mathrm{~nm}, 6 \mathrm{~mW} / \mathrm{cm}^{2}\right)$.

\section{Materials and Methods}

Firstly, the $\mathrm{n}$-Si (111) substrates were held in high vacuum (10-6 Torr) using a rapid thermal chemical vapor deposition (RTCVD) system at $900{ }^{\circ} \mathrm{C}$ for $10 \mathrm{~min}$ to remove the native oxide layer. Then, the substrates were cooled to room temperature. Subsequently, the n-Si (111) substrates were carbonized with propane $\left(\mathrm{C}_{3} \mathrm{H}_{8}\right) \cdot{ }^{(6,7)}$ After that, the substrate temperature was rapidly increased to 1000-1200 ${ }^{\circ} \mathrm{C}$, and then, $\mathrm{SiH}_{4}(85 \mathrm{sccm}), \mathrm{C}_{3} \mathrm{H}_{8}(60 \mathrm{sccm})$, and $\mathrm{H}_{2}(50 \mathrm{sccm})$ gases were introduced into the reaction chamber and the pressure was kept at $5 \mathrm{mTorr}$ to grow $\beta$-SiC films. The growth rate was about $200 \AA / \mathrm{min}$. For the buffer application, the $\beta$-SiC films were grown to have a thickness of about $6000 \AA$. On the other hand, to form PSC layers, the deposited $\beta$-SiC films were etched in a solution ( $\left.\mathrm{HF}: \mathrm{H}_{2} \mathrm{O}: \mathrm{C}_{2} \mathrm{H}_{5} \mathrm{OH}=1: 1: 2\right)$ under conditions of $1.9 \mathrm{~V}, 2 \mathrm{~mA} / \mathrm{cm}^{2}$ current density, and illumination by a $50 \mathrm{~W}$ halogen lamp for $2 \mathrm{~min} \cdot{ }^{(8)}$

Next, the GaN films were grown on the c-SiC/Si or PSC/Si substrates in a Thomas Swan designed MOCVD reactor. First, the c-SiC/Si and PSC/Si substrates were cleaned and immediately placed into the reactor. Then, the 1 st $500 \AA \mathrm{GaN}$ layer was grown at $550{ }^{\circ} \mathrm{C}$ under the following conditions: 300 Torr for the reactor pressure, $100 \mathrm{rpm}$ for the substrate rotation speed, $10000 \mathrm{sccm}$ for the flow rate of ammonia $\left(\mathrm{NH}_{3}\right)$ gas, $28 \mathrm{sccm}$ for trimethylgallium (TMGa) gas, and 10000 sccm for carrier gas $\mathrm{H}_{2}$. The growth rate of the $1 \mathrm{st} \mathrm{GaN} \mathrm{layer} \mathrm{was} \mathrm{about} 120 \AA / \mathrm{min}$. Next, a $3 \mu \mathrm{m}$ 2nd GaN film was grown sequentially at $1100{ }^{\circ} \mathrm{C}$ with the conditions of 600 Torr and $100 \mathrm{rpm}$. The flow rates of $\mathrm{NH}_{3}$ gas, $\mathrm{TMGa}$, and carrier gas $\mathrm{H}_{2}$ were 12000,85 , and $10000 \mathrm{sccm}$, respectively. The growth rate of the $2 \mathrm{nd} \mathrm{GaN}$ layer was about $167 \AA / \mathrm{min}$. Finally, Au was evaporated onto the top of the 2 nd GaN layer to form finger-mask electrodes to obtain MSM GaN photodetectors.

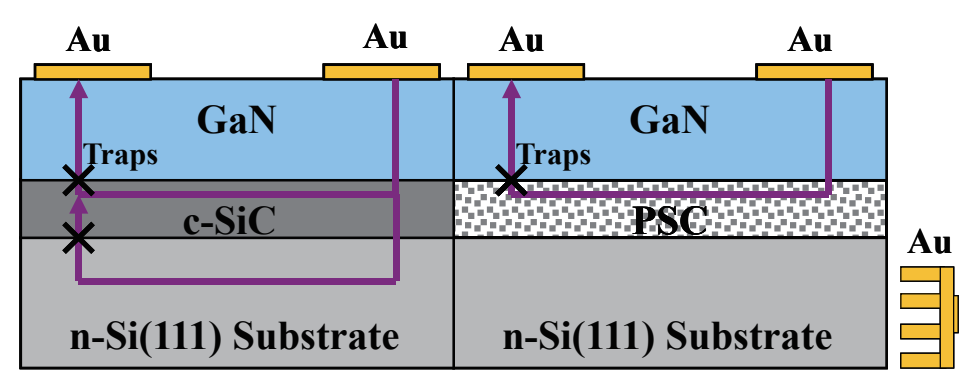

Fig. 1. (Color online) Schematic diagram of the GaN MSM photosensing devices fabricated on the c-SiC/n-Si and PSC/n-Si substrates. The arrows indicate the possible current paths. 


\section{Results}

Figure 2(a) shows X-ray diffraction (XRD) spectra of the $\beta$-SiC(111) films grown on the $\mathrm{n}-\mathrm{Si}$ (111) substrate obtained at various growth temperatures from 1000 to $1200{ }^{\circ} \mathrm{C}$. At $1000{ }^{\circ} \mathrm{C}$, the grown film appears as an amorphous structure and no obvious peaks are found. At $1100{ }^{\circ} \mathrm{C}$, the film becomes a polycrystalline structure with peaks appearing at $2 \theta=35.8,59.86$, and $72.053^{\circ}$ for $\mathrm{SiC}$ (111), $\mathrm{SiC}(220)$, and $\mathrm{SiC}$ (311), respectively. As the temperature increases to $1200^{\circ} \mathrm{C}$, the film turns into crystalline $\beta-\mathrm{SiC}$, and thus the peak appears only at $2 \theta=35.8^{\circ}$. On the basis of Fig. 2(a) and Scherrer's formula $\left[D_{\text {(hkl) }}=0.9 \lambda /(\beta \cos \theta)\right],{ }^{(9,10)}$ the crystal grain sizes of the as-grown $\beta$-SiC (111) films are 11.7 and $14.0 \mathrm{~nm}$ for 1100 and $1200^{\circ} \mathrm{C}$, respectively. Here, $D_{\mathrm{hkl}}$ is the grain size, $\lambda(=0.1514$ $\mathrm{nm}$ ) is the $\mathrm{X}$-ray wavelength, $\beta$ is the full width at half maximum measured from the $\mathrm{SiC}$ (111) peak at the growth temperature, and $\theta\left(=35.8^{\circ} / 2\right)$ is Bragg's angle at the c-SiC (111) peak. The crystal grain size increases with increasing growth temperature in the two-dimensional growth modes and dominates in $\mathrm{SiC}$ growth ${ }^{(9)}$ indicating that the crystal quality of the $\mathrm{SiC}$ films can be improved by adjusting the growth temperature.

In addition, the corresponding Fourier transform infrared spectroscopy (FTIR) spectra of these samples are presented in Fig. 2(b) to analyze the bonding structure between $\mathrm{Si}$ and $\mathrm{C}$ at various growth temperatures. As seen, the peak of the $\mathrm{Si}-\mathrm{C}$ bond (i.e., the number of $\mathrm{Si}-\mathrm{C}$ bonds) appears at $794.6 \mathrm{~cm}^{-1}$ and increases with growth temperature from 1000 to $1200{ }^{\circ} \mathrm{C}$, meaning that a high growth temperature is suitable for the growth of a crystalline film. Moreover, the measured peak of the Si-C bond for a wave number of $794.6 \mathrm{~cm}^{-1}$ is slightly lower than that for $796.2 \mathrm{~cm}^{-1}$ for the defect-free $\beta$-SiC film on $\mathrm{n}-\mathrm{Si}(111),{ }^{(10,11)}$ thus implying the existence of stress in the $\beta$-SiC film grown on $\mathrm{n}-\mathrm{Si}$ (111). The stress contributes to the difference between the thermal expansion coefficient of $\mathrm{Si}$ and that of $\beta$-SiC. Both Figs. 2(a) and 2(b) indicate that the $\beta$-SiC films grown on the $\mathrm{n}-\mathrm{Si}$ (111) substrate at 1000 and $1100{ }^{\circ} \mathrm{C}$ are still amorphous and polycrystalline, respectively. To obtain a crystalline $\mathrm{SiC}$ film, the temperature should be raised to $1200^{\circ} \mathrm{C}$ or over.

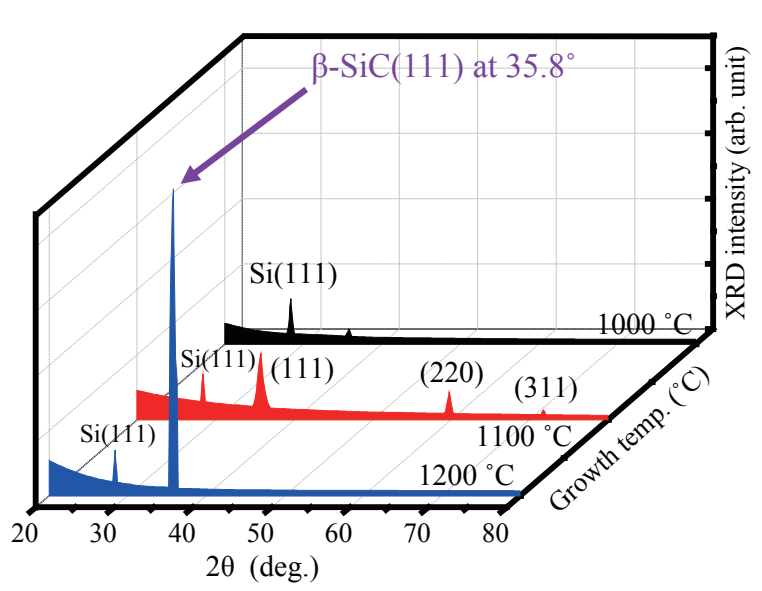

(a)

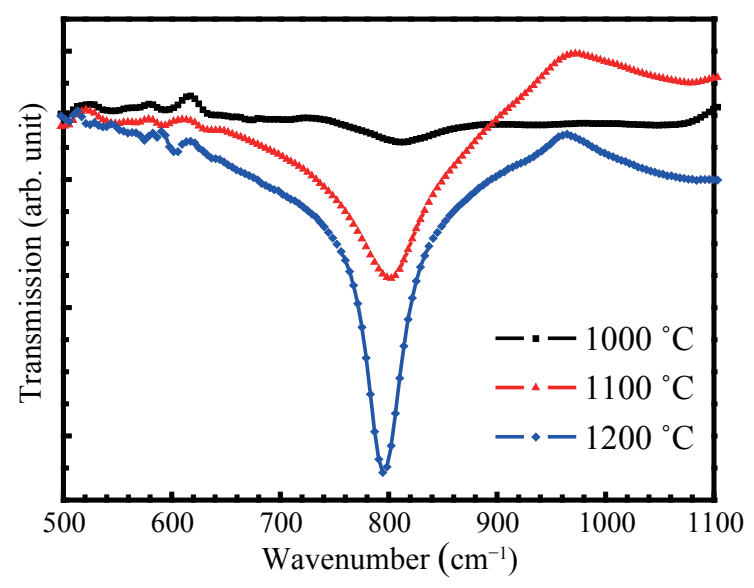

(b)

Fig. 2. (Color online) (a) XRD spectra for the $\beta$-SiC films on the $\mathrm{Si}$ substrate at various growth temperatures of $1000-1200{ }^{\circ} \mathrm{C}$. (b) FTIR spectra for the SiC films at various growth temperatures of $1000-1200{ }^{\circ} \mathrm{C}$. 
We also used atomic force microscopy (AFM) to examine the morphology of the films as shown in Figs. 3(a)-3(c). As the growth temperature increases, the grain size increases and thus, the surface roughness $(R a)$ increases to reach the maximum (5.17 and $7.034 \mathrm{~nm}$ for $R a$ and rootmean-square roughness, respectively) at $1200{ }^{\circ} \mathrm{C}$. Figure 4 shows side-view scanning electron microscopy (SEM) images of the GaN films deposited on the (a) PSC/n-Si (111) and (b) c-SiC/n-Si (111) substrates. The GaN film grown on the PSC/n-Si (111) substrate is denser and has a more regular columnar structure than that grown on the c-SiC/n-Si (111) substrate.

Raman spectra of the GaN/PSC/n-Si and GaN/c-SiC/n-Si at room temperature are presented in Fig. 5. The dominant $E_{2}$ (high) phonon mode appears at 567.9 and $571.3 \mathrm{~cm}^{-1}$. Moreover, the $\mathrm{E}_{2}$ phonon mode of bulk single-crystal GaN is shown at $566.2 \mathrm{~cm}^{-1}$ as the reference for an unstressed film. . $^{(12)}$ The biaxial stress $\sigma_{\mathrm{xx}}$ in the GaN film can be calculated from the relation $\sigma_{\mathrm{xx}}=\left(\Delta \omega_{\gamma} / K_{\gamma}\right){ }^{(13)}$ where $\sigma_{\mathrm{xx}}$ is the stress in GPa, $\Delta \omega_{\gamma}$ is the Raman shift in $\mathrm{cm}^{-1}$, and $K_{\gamma}$ is the stress coefficient (4.3 $\left.\mathrm{cm}^{-1} \mathrm{GPa}^{-1}\right)$. However, the biaxial strain originating from the growth on the lattice-mismatched substrate and from the difference in the thermal expansion coefficients of the GaN film and the n-Si (111) substrate introduced a stress (blue shift) to generate cracks in the brittle material.(12) The $\sigma_{\mathrm{xx}}$ values of the $\mathrm{GaN} / \mathrm{PSC} / \mathrm{n}-\mathrm{Si}$ and $\mathrm{GaN} / \mathrm{c}-\mathrm{SiC} / \mathrm{n}-\mathrm{Si}$ samples are 0.395 and $1.186 \mathrm{GPa}$, respectively. The $\sigma_{\mathrm{xx}}$ value of the GaN/PSC/n-Si sample is only $33.3 \%$ of that of the GaN/c-SiC/n-Si sample. Since the

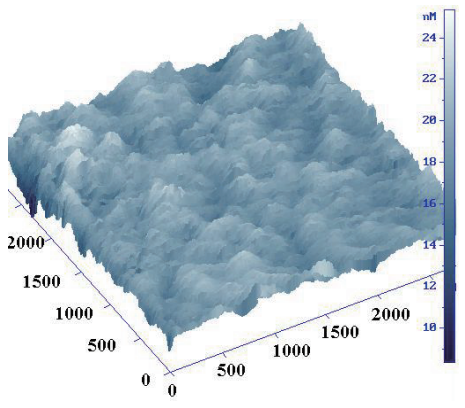

(a)

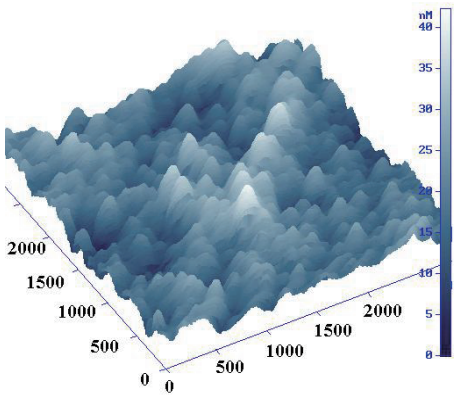

(b)

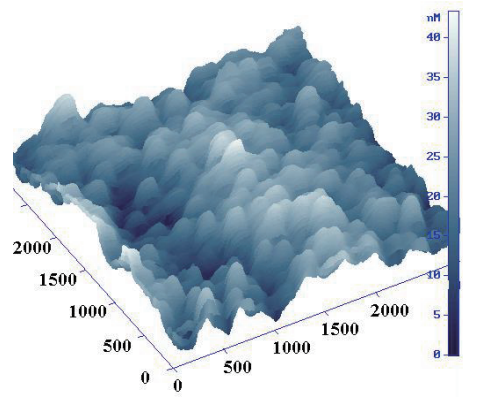

(c)

Fig. 3. (Color online) AFM images of as-deposited $\mathrm{SiC} / \mathrm{Si}$ films at various growth temperatures of $1000-1200{ }^{\circ} \mathrm{C}$. (a) $R a=1.098 \mathrm{~nm}$ and $R M S=2.031 \mathrm{~nm}$, (b) $R a=4.435 \mathrm{~nm}$ and $R M S=5.142 \mathrm{~nm}$, and (c) $R a=5.170 \mathrm{~nm}$ and $R M S=7.034$ $\mathrm{nm}$.

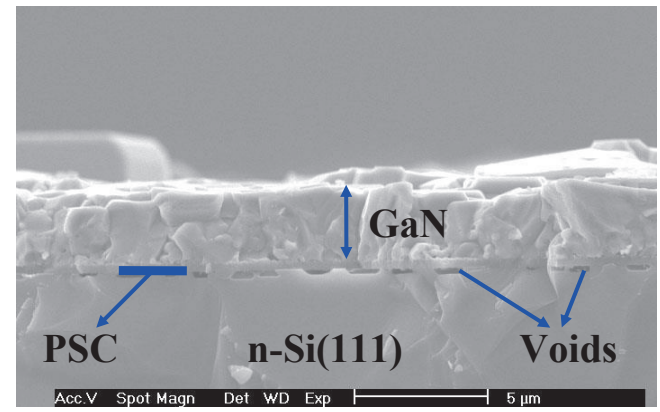

(a)

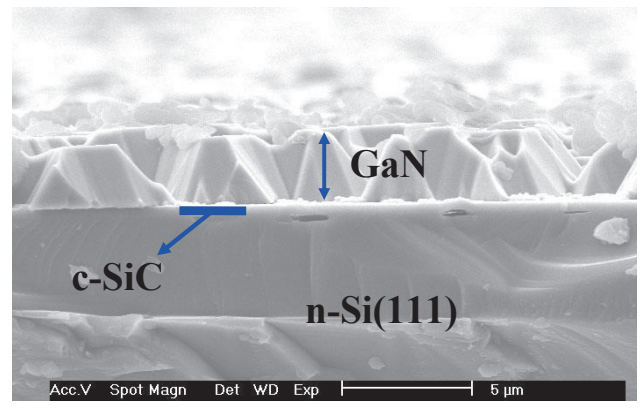

(b)

Fig. 4. (Color online) Side-view SEM images of the as-deposited (a) GaN/PSC/n-Si (111) and (b) GaN/c-SiC/ n-Si (111). 


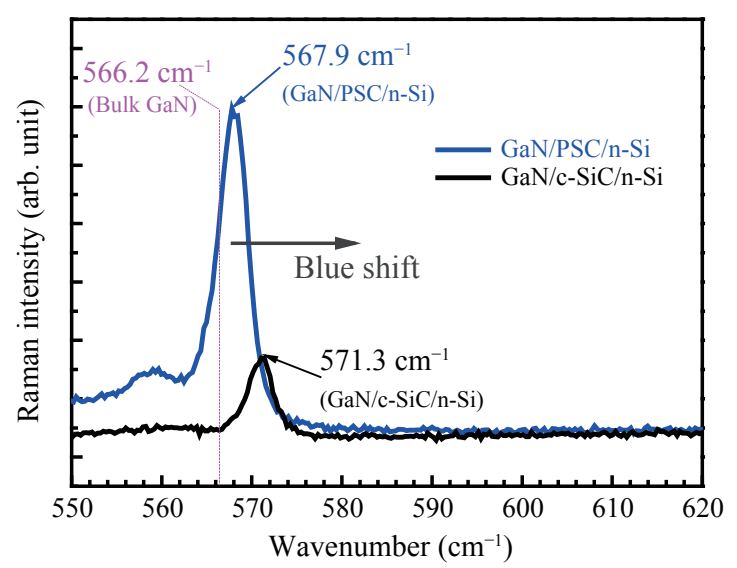

Fig. 5. (Color online) Raman spectra measured at room temperature on the GaN/PSC/n-Si and GaN/ c-SiC/n-Si samples obtained using $325 \mathrm{~nm} \mathrm{He}-\mathrm{Cd}$ pumping laser.

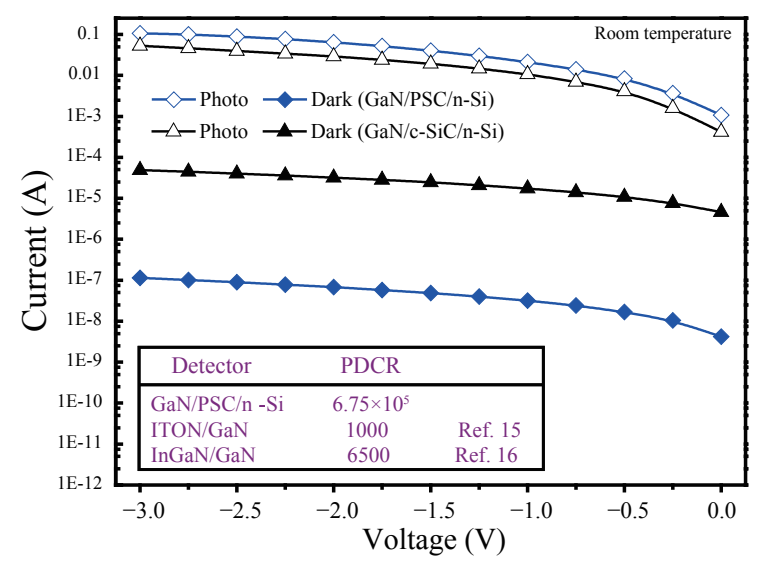

Fig. 6. (Color online) Dark current and photocurrent for the GaN/PSC/n-Si and GaN/c-SiC/ n-Si photodetectors.

stress is low, the high-quality GaN thin film can be grown on the n-Si (111) substrate with a PSC film as the buffer layer.

Finally, the room-temperature dark current and photocurrent of the GaN/PSC/n-Si and $\mathrm{GaN} / \mathrm{c}-\mathrm{SiC} / \mathrm{n}$-Si MSM photodetectors were measured under reversed biases with an HP4145B semiconductor parameter analyzer, as shown in Fig. 6. The dark current of the GaN/PSC/n-Si photodetector was much lower than that observed from the GaN/c-SiC/n-Si photodetector. This is attributed to the reduction of dislocation density (see Fig. 1) and the value of $\sigma_{\mathrm{xx}}$ from the PSC buffer layer, which might lead to higher stress relaxation. ${ }^{(12-14)}$ The photocurrents were measured under irradiation by a $366 \mathrm{~nm}$ UV light source (Model: UVP, UVGL-58) with $6 \mathrm{~mW} / \mathrm{cm}^{2}$ power.

On the basis of the measured currents, the PDCR is calculated using the formula PDCR $=\left[\left(I_{\mathrm{p}}\right.\right.$ $\left.\left.-I_{\mathrm{d}}\right) / I_{\mathrm{d}}\right]$, where $I_{\mathrm{d}}$ is the dark current and $I_{\mathrm{p}}$ is the photocurrent (i.e., the current under illumination). The room-temperature PDCR under $-1 \mathrm{~V}$ bias is $6.75 \times 10^{5}$ for GaN/PSC/n-Si, which is better than the reported $\sim 1000$ for an ITON/GaN photodetector, ${ }^{(15)}$ or $\sim 6500$ for an $\mathrm{InGaN} / \mathrm{GaN}$ MOW photodetector ${ }^{(16)}$ as compared in the inset of Fig. 6 . These results imply that the large PDCR could be attributed mainly to the fact that the PSC buffer layer can reduce $\sigma_{\mathrm{xx}}$ and the dislocation density (see Fig. 1), leading to the growth of the high-quality GaN film deposited on the n-Si (111) substrate.

\section{Conclusions}

High-quality GaN films for UV-detecting applications were grown by MOCVD on n-Si (111) substrates using c-SiC and PSC as buffer layers. Even though the PSC buffer layer on Si had the roughest surface morphology, its spongelike structure relieved the development of strain and cracks after the GaN film was grown to obtain the smallest stress between the GaN film and the $\mathrm{n}-\mathrm{Si}$ (111) substrate. Therefore, the GaN films grown on the PSC/n-Si buffer layer had the best film morphology and structure to achieve the highest UV optoelectronic performance. Therefore, we conclude that the reduction of the stress built in the GaN films is the key technology to successful heteroepitaxial growth of $\mathrm{GaN}$ on $\mathrm{Si}$ substrates. 


\section{References}

1 J. Napierala, H.-J. Bühlmann, and M. Ilegems: J. Electrochem. Soc. 153 (2006) G125.

2 F. A. Ponce, B. S. Krusor, J. S. Major, Jr., W. E. Plano, and D. F. Welch: Appl. Phys. Lett. 67 (1993) 410.

3 D. Wang, Y. Hiroyama, M. Tamura, M. Ichikawa, and S. Yoshida: Appl. Phys. Lett. 77 (2000) 1846.

4 H. Hartono, C. B. Soh, S. J. Chua, and E. A. Fitzgerald: J. Electrochem. Soc. 154 (2007) H1004.

5 Y. Nakada, I. Aksenov, and H. Okumura: Appl. Phys. Lett. 73 (1998) 827.

6 B. Burkland, Z. Y. Xie, J. H. Edgar, M. Ervin, J. Chaudhuri, and S. Farsinivas: J. Electrochem. Soc. 149 (2002) G550.

7 K. H. Wu, Y. K. Fang. J. H. Zhou, and J. J. Ho: Jpn. J. Appl. Phys. 36 (1997) 5151.

8 A. Takazawa, T. Tamura, and M. Yamada: Jpn. J. Appl. Phys. 32 (1993) 3148.

9 W. V. Muench and E. Pettenpaul: J. Electrochem. Soc. 125 (1978) 294.

10 K. C. Kim, C. I. Park, J. I. Roh, K. S. Nahm, Y. B. Hahn, Y. S. Lee, and K. Y. Lim: J. Electrochem. Soc. 148 (2001) C383.

11 H. Mukaida, H. Okumura, J. H. Lee, H. Daimon, E. Sakuma, S. Misawa, K. Endo, and S. Yoshida: J. Appl. Phys. 62 (1987) 254.

12 C. Kisielowski, J. Krüger, S. Ruvimov, T. Suski, J. W. Ager III, E. Jones, Z. L. Weber, M. Rubin, E. R. Weber, M. D. Bremser, and R. F. Davis: Phys. Rev. B 54 (1996-II) 17745.

13 D. G. Zhao, S. J. Xu, M. H. Xie, S. Y. Tong, and H. Yang: Appl. Phys. Lett. 83 (2003) 677.

14 A. Sagar, C. D. Lee, R. M. Feenstra, C. K. Inoki, and T. S. Kuan: J. Vac. Sci. Technol. B 21 (2003) 1812.

15 N. Vanhove, J. John, A. Lorenz, K. Cheng, G. Borghs, and J. E. M. Haverkort: Appl. Surf. Sci. 253 (2006) 2930.

16 Y. Z. Chiou, Y. K. Su, S. J. Chang, Y. C. Lin, C. S. Chang, and C. H. Chen: Solid State Electron. 46 (2002) 2227. 